\title{
DEEP GALAXY COUNTS AND COSMOLOGY
}

\author{
Masataka Fukugita \\ Yukawa Institute for Theoretical Physics, Kyoto University \\ Kyoto 606, Japan
}

The test of the world geometry with the number count of galaxies is one of the most classical subjects. Yet it is only quite recent that observations started to yield useful information on the world geometry (Tyson 1988). We have been studying this cosmology test over the last few years (Fukugita et al. 1990; see also Yoshii and Takahara 1988), recently with more emphasis given to galaxy evolution and selection effects of the observation. In this talk we summarise the main result from our study (Fukugita et al. 1991; Yoshii and Fukugita 1991b).

We assume that total numbers of galaxies of each morphological type are conserved separately. We also assume that evolution takes place only through the change of the characteristic luminosity $L_{i}^{*}$, which, however, may depend on the morphological type $i$. The population synthesis model of Arimoto and Yoshii (1987) is adopted to predict the evolution of $L_{i}^{*}$. We take the Schechter luminosity function with its parameters assumed to be universal at the present epoch.

Our results are:

1) Luminosity evolution is necessary for $B_{J} \gtrsim 21 \mathrm{mag}$. The number count up to $B_{J} \approx 24 \mathrm{mag}$ is described reasonably well with our fiducial evolution model.

2 ) The B band counting (Tyson 1988) is fitted well with the $\Lambda$-dominated cosmology $\left(\Omega_{0}=0.1, \lambda_{0}=0.9\right)$ beyond $B_{J} \approx 24 \mathrm{mag}$. At a more quantitative level the observed count lies dex 0.2 above the prediction throughout $23-27 B_{J}$ mag. The low massdensity model $\left(\Omega_{0}=0.1, \lambda_{0}=0\right)$ predicts too small a number of galaxies at deep magnitudes. This prediction, however, might be modified, if one would make an evolution model where $u v$ in the past is stronger than that in the present model. The $\Omega_{0}=1$ cosmology is difficult to reconcile with the observation, since the predicted $N(m)$ flattens at around $26 \mathrm{mag}$ and the value at this plateau is significantly (dex 0.7 ) lower than the observation (Fukugita et al. 1990).

3) Basically the same conclusion is derived from the R and I band counts of Tyson: The best fit is obtained with the $\Lambda$ - dominated cosmology $\left(\Omega_{0}=0.1, \lambda_{0}=0.9\right)$. The $\Omega_{0}=1$ cosmology is strongly disfavoured.

4) The K-band count (Cowie et al. 1990; 1991) is fitted best with the low density 
cosmology $\left(\Omega_{0}=0.1, \lambda_{0}=0\right)$; see Fig. 1 . The $\Omega_{0}=1$ cosmology predicts $N(m)$ significantly short of the observed count. The prediction of the $\Lambda$-dominated cosmology overshoots the data. This may be taken to suggest a problem with the closed evolution model: The K-band count is sensitive to the old population. If stars form gradually in the first Gyrs, as in the case when gas infall plays an important role, galaxies are fainter in the $\mathrm{K}$-band than are expected from the galaxy today in the present model. For a different view point, see Cowie et al. 1991, however.

5) A study is made on the possibility that faint galaxies seen by Tyson are intrinsically faint objects (dwarfs) placed not at a great distance. In fact the Tyson's counting may be fitted well with any cosmology, if the dwarf component is more important (i.e., $\alpha=-2$, say) than is expected from the local luminosity function. In this case, however, the redshift distribution $N(z)$ shows a gross disagreement with the observation of the Durham survey (Broadhurst et al. 1988; Colless et al. 1991). Therefore, this possibility is not likely.

For the colour distribution we see that:

6) A gross agreement is seen between the prediction and observations in the B-R colour distribution. At a more quantitative level, however, systematic disagreements exist; (i) the observed distribution extends to the bluer side than is predicted and (ii) a spurious red peak emerges in the prediction in fainter magnitudes. We consider, however, these fine structures disturb little the prediction of the number count.

In our study we also found that selection effects of the observation are often crucial in interpreting the data. Specifically, we are concerned with (i) the effect of the use of isophotal magnitudes to define brightness, (ii) finite seeing, and (iii) the galaxy selection criterion defined by the minimum size of images. We found:

7) Effect (i) plays a crucial role in the interpretation of $N(z)$. The use of isophotal magnitudes leads to a systematic neglect of higher $z$ galaxies. In fact models with canonical evolution fit very well the Durham data if the isophotal effect is taken into account, especially with the low density model. No-evolution models give too little galaxies for the higher $z$ side of the $N(z)$ distribution (Yoshii and Fukugita 1991a). 8) A sharp cut-off of $N(m)$ at the faintest magnitude is ascribed to (iii), and (ii) which softens the cut-off a little. The $\Lambda$-dominated model $\left(\Omega_{0}=0.1, \lambda_{0}=0.9\right)$ reproduces the Tyson's raw data very well for the entire magnitude ranges for the B, R and I bands (e.g., Fig.2).

9) A simple merging hypothesis can not save the $\Omega_{0}=1$ model. If we require that the total luminosity of galaxies be conserved through merging and that the star luminosity density of premerger galaxies be equal to or larger than the postmergers, we can show that most of premergers should have been missed in the Tyson's observation due to (iii). Hence, even if the nominal number of galaxies increases towards high $z$ as $(1+z)^{\gamma}$ with $\gamma \sim 4-5$, the observable index remains very close to $\gamma=3$; $N\left(B_{J}\right)$ increases only by dex 0.2 at $B_{J}=27 \mathrm{mag}$ for $\gamma \sim 4-6$.

I would like to thank Lennox Cowie for discussions. I am also grateful to the Yamada Science Foundation for the support. 


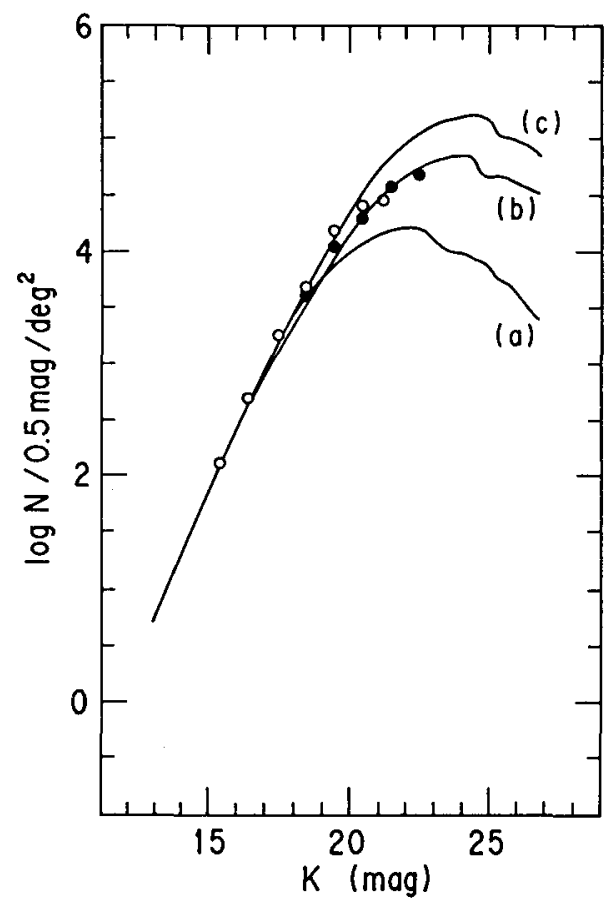

Fig.1 The $K$-band count of Cowie et al. $(1990,1991)$ is compared with the model predictions for three cosmologies: (a) $\Omega_{0}=1$, (b) $\Omega_{0}=0.1$, (c) $\Omega_{0}=0.1, \lambda_{0}=0.9$.

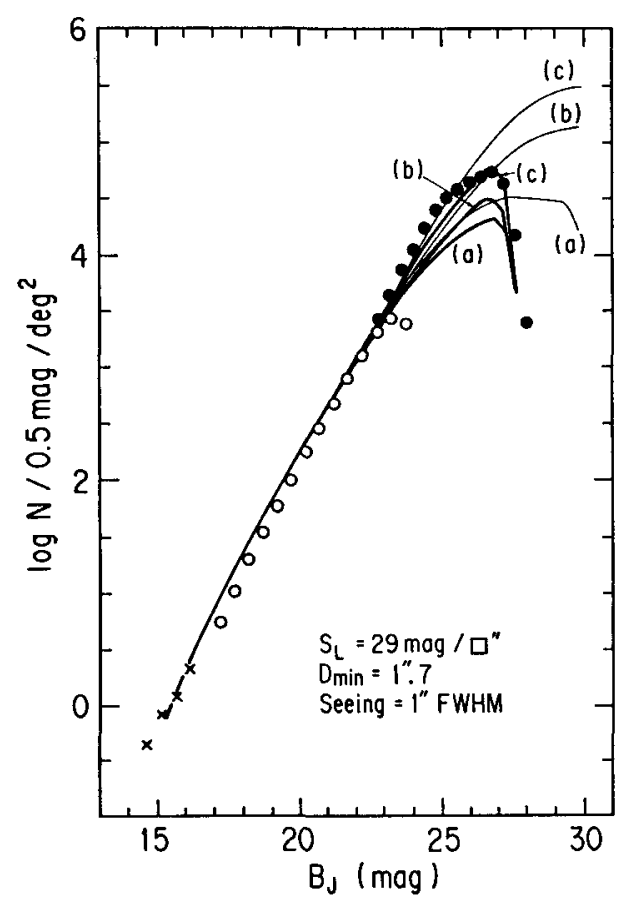

Fig.2 The predictions for $N(m)$ with the selection effects are compared with the raw data by Tyson (1988). Thin curves are predictions without the selection effects.

\section{References}

Arimoto, N. \& Yoshii, Y. 1986, Astr. Astrophys. 164, 260.

Broadhurst, T.J., Ellis, R.S. \& Shanks, T. et al. 1988, MNRAS 235, 827.

Colless, M. et al. 1991, MNRAS 244, 408.

Cowie, L.L. et al. 1990, Astrophys. J. 360, L1.

$$
\text { 1991, preprint. }
$$

Fukugita, M., Takahara, F., Yamashita,K. \& Yoshii,Y. 1990, Astrophys. J. 361,L1. 1991, in preparation.

Tyson, J.A. 1988, Astron. J. 96, 1.

Yoshii, Y. \& Fukugita, M. 1991a, in Observational Test of Inflation, in press.

Yoshii, Y. \& Fukugita, M. 1991b, in preparation.

Yoshii, Y. \& Takahara, F. 1988, Astrophys. J. 326, 1. 